\title{
Endogenous locus-driven H-Ras G12V expression induces senescence-like phenotype in primary fibroblasts of the Costello syndrome mouse model
}

\author{
Saravanakkumar Chennappan ${ }^{1}$, Tanja Schulze ${ }^{1}$, Ion Cristian Cirstea ${ }^{1,2, *}$ \\ ${ }^{1}$ Leibniz Institute on Aging, Beutenberg Str. 11, 07745 Jena, Germany \\ ${ }^{2}$ Institute of Comparative Molecular Endocrinology (CME), Ulm University, Helmholtz Str. 8/1, 89081 Ulm, Germany
}

(Received 9 March, 2017; accepted 23 April, 2017)

\begin{abstract}
Oncogenic H-Ras germline mutations are associated with Costello syndrome (CS), a developmental disorder characterised by the failure to thrive, skin and musculoskeletal abnormalities, cardiomyopathy, and susceptibility to tumour development. Despite harbouring H-Ras oncogenic mutations, in both patients and a CS mouse model, the tumour incidence is very low. Using CS mouse primary fibroblasts, we identified that unlike the lack of tumorigenic transformation, the MAPK and Akt pathways display subtle changes in their activation kinetics. In vivo, these subtle changes might not be sufficient to induce malignant transformation, but may well be responsible for inducing CS pathologies. Moreover, we identified an oncogene-induced senescence-like phenotype, and we consider it as a potential mechanism in order to block tumour development in CS. The induction of oncogene-induced senescence-like phenotype can ultimately affect proliferative cells (e.g., progenitor cells) and impair tissue homeostasis during embryonic development and throughout the life span of CS patients.
\end{abstract}

Keywords: endogenous, H-Ras, rasopathies, Costello syndrome, oncogene-induced senescence, tumour suppression, pathologies

\section{INTRODUCTION}

Rasopathies are developmental disorders arising from mutations in genes encoding for Ras-MAPK pathway components (1). One of these is Costello syndrome (CS), a rare disorder resulting from germline mutations in the $H$-Ras oncogene. Young patients display craniofacial, skin, musculoskeletal, and cardiac abnormalities as well as a predisposition to develop tumours (2). During the aging process, patients develop osteoporosis, hypertrophic cardiomyopathies, a reduced muscle tone and age-related cancer (e.g., bladder cancer) (3). An interesting aspect of the patients' pathology is that, despite harbouring an oncogenic mutation in virtually every cell of the body, only approximately $10 \%$ of the CS patients develop tumours such as bladder cancer, rhabdomyosarcoma or neuroblastoma (4). H-Ras is a molecular switch, which controls many signalling pathways leading to proliferation, differentiation, migration, and apoptosis, among other biological processes $(5,6)$. It cycles between an inactive form (GDP-bound) and an active form (GTP-bound), and this is regulated by two classes of proteins: guanine nucleotide exchange factors (GEFs), which accelerate activation via GDP for GTP exchange; and GTPase-activating proteins (GAPs), which catalyse GTP hydrolysis and inactivation (7). Oncogenic somatic mutations in the H-Ras gene affect GAP-mediated hydrolysis and keep H-Ras in a constitutively active state $(8,9)$, which in turn leads to hyper-activation of its downstream pathways. In CS, germline mutations affect similar residues as the somatic oncogenic mutations, with the Gly12 to serine (G12S) substitution identified in more than $80 \%$ of patients. Other mutations (e.g., G12V, G12D) are rare, and the affected patients usually develop a stronger phenotype which results in postnatal fatality (10-12).

Research on CS was advanced by the creation of a mouse model harbouring an $\mathrm{H}-\mathrm{Ras} \mathrm{G} 12 \mathrm{~V}$ germline mutation. This mouse phenocopies some features of the CS patients: craniofacial dysmorphia, skeletal abnormalities, hypertrophic cardiomyopathy, neurological and behavioural alterations $(13,14)$. Using another mouse model harbouring the same mutation, an essential role for H-Ras in tooth development was established (15). At the same time, a combination of in vitro and in vivo studies (using in utero electroporation of embryos), proved that neurogenesis is impaired by H-Ras mutations in cortical progenitor cells. By favouring premature gliogenesis over

*Corresponding author (E-mail: ion.cirstea@uni-ulm.de) 
neurogenesis, H-Ras mutations dramatically increased the number of astrocytes (16).

So far, most of the studies have focused either on mutational identification or on a phenotypic description and the identification of biological processes underlying CS pathologies, and not so much on the molecular basis of dysregulated H-Ras signalling. In the H-Ras G12V mouse model, a basic analysis of activation and its downstream signalling network identified that in spite of the constitutive activation of $\mathrm{H}$-Ras, there is no increase in the activation of MAPK and Akt pathways in both primary cells (cardiomyocytes, mouse embryonic fibroblasts) and organs (heart, kidney) (14). Because of the fact that they harbour an oncogenic mutation, CS mice were surveyed for tumour induction during their life span. CS mice did not develop spontaneous tumours and were resistant to carcinogen-induced tumour development.

In this study, using mouse embryonic fibroblasts (MEFs) and adult skin fibroblasts (ASFs) derived from the same mouse model, we have performed a deeper in vitro molecular analysis of $\mathrm{H}-\mathrm{Ras}$ signalling. We aimed to identify the mechanisms responsible for a reduced activation of $\mathrm{H}$-Ras downstream pathways in spite of $\mathrm{H}$-Ras constitutive activation and to monitor the changes in the epidermal growth factor (EGF)-mediated activation kinetics of MAPK and Akt pathways, two hallmarks for the dysregulated signalling which leads to CS pathologies. In addition to MEFs, by using the adult ASFs cell model, we tested the activation of both pathways in basal conditions or in response to growth factor stimulation. Furthermore, we monitored cellular proliferation and the expression of cell cycle inhibitory genes, p16ink4a and p2lwaf, as modulators of oncogene-induced senescence (OIS) in CS ASFs. In CS MEFs, we noticed that the expression of the Erk1/2 inhibitory protein, dual specificity phosphatase 6 (Dusp6), the tumour suppressors, phopsho-p53 and phospho-Rb, and the proliferation marker, proliferating cell nuclear antigen (PCNA) did not increase in response to oncogenic H-Ras. Additionally, CS MEFs activation kinetics analysis showed subtle changes in Erk1/2 and Akt activation in response to EGF induction. Finally, in ASFs H-Ras G12V mutations induced Akt pathway upregulation under basal stimulation, slowed down cellular proliferation, triggered an upregulation in gene expression of cell cycle inhibitory proteins ( $p 16 i n k 4 a, p 21 w a f)$, and led to an increased reactive oxygen species production. These later features imply a potential induction of oncogene-induced senescence (OIS), which could be the mechanism deterring malignant transformation in our CS cellular model, and presumably in CS mouse model. On the other hand, the induction of OIS-like phenotype due to changes in the signalling pathways triggered by oncogenic H-Ras may well be another molecular mechanism which leads to CS dysregulated signalling and pathologies.

\section{MATERIAL AND METHODS}

\section{Mice}

H-Ras G12Vgeo mice were a kind gift from Prof. Mariano Barbacid (CNIO, Spain), and were backcrossed and maintained in the $\mathrm{C} 57 \mathrm{BL} / 6$ background. All animal usage for the purpose of embryo and tissue collection was strictly within the guidance set by the institutional animal care policy and authorised by the local animal welfare authorities.

\section{Mouse embryonic fibroblasts (MEFs) isolation and sub-culturing}

Embryos for MEF isolation were obtained from timely paired heterozygous female to a heterozygous male. At day 13.5 post coitus, the pregnant females were sacrificed by $\mathrm{CO}_{2}$ inhalation, and the uterus with embryos was removed, washed once in 70\% ethanol and placed in a $10 \mathrm{~cm}$ dish with sterile $\mathrm{PBS}^{++}$(Invitrogen \# 14040). The embryos were separated from the placenta and surrounding membranes, and transferred to individual $10 \mathrm{~cm}$ dishes containing sterile $\mathrm{PBS}^{++}$. Foetal livers were removed, and the heads were placed in micro centrifuge tubes for genomic DNA isolation for genotyping. The embryo body was minced and digested with $3 \mathrm{~mL}$ of digestion buffer containing $0.1 \%$ Collagenase Type I (Merck Millipore) and $2.4 \mathrm{U} / \mathrm{mL}$ Dispase II (Sigma-Aldrich) in DMEM/F12 (Invitrogen) supplemented with $1 \%$ antibiotics. Digestion was carried out with mild stirring on a magnetic stirrer, placed in a cell culture incubator, until the tissue dissociated into a cellular suspension. After digestion, the cells were pelleted and washed twice with pre-warmed MEF culture media containing DMEM/F12, 10\% FBS (Sigma-Aldrich), $1 \%$ Penicillin-Streptomycin (Sigma-Aldrich) and 1\% Antibiotic Antimycotic (Sigma-Aldrich) to remove Dispase II and collagenase. The final pellet was re-suspended in $13 \mathrm{~mL}$ MEF culture media and was seeded in to a T75 flask. On day 2, the cells were trypsinised and sub-cultured (designated as passage 1, P1) using a modified 3T3 protocol which was optimised for efficient MEF maintenance (17). Every third day, the cells were detached, counted and re-seeded at a density of $0.7 \times 10^{6}$ cells $/ 10 \mathrm{~cm}$ cell culture dish. MEFs were routinely cultured in $\mathrm{MEF}$ culture media at $37^{\circ} \mathrm{C}$ in a $21 \% \mathrm{O}_{2}$ (atmospheric $\mathrm{O}_{2}$ ) and $5 \% \mathrm{CO}_{2}$ incubator.

\section{Mouse adult skin fibroblasts (ASFs) isolation and sub-culturing}

Adult mouse primary skin fibroblasts (ASF) were isolated from 6-7-month-old female mice. The area of skin covering the under arm and over the rib cage was used for fibroblast isolation. The shaved surface was sterilized with $70 \%$ ethanol and the skin was removed and placed into a sterile $50 \mathrm{~mL}$ falcon containing DMEM/ F12 media and 1\% antibiotics and stored on ice. Once 
all the samples were collected, the excised skins were spread dermis side down into a sterile well of a 6 well tissue culture dish containing 2,4 U/mL Dispase II in $\mathrm{PBS}++$ and were incubated overnight at $4^{\circ} \mathrm{C}$. Following the removal of epidermis and excess fat, the dermis was chopped into small pieces and incubated with digestion media at $37^{\circ} \mathrm{C}$ on a magnetic stirrer, placed in a cell culture incubator, and incubated until the chopped tissue was easy to pipette or until the tissue dissociated into a cellular suspension. Cells were pelleted and washed twice with pre-warmed ASF culture media containing DMEM-F12, 10\% FBS, 1\% Penicillin-Streptomycin, 1\% Antibiotic Antimycotic, $10 \mathrm{ng} / \mathrm{mL}$ bFGF (ImmunoTools) and $0.4 \mu \mathrm{M} \quad \beta$-mercaptoethanol (Invitrogen) to remove Dispase II and collagenase. The final pellet was re-suspended in $6 \mathrm{~mL}$ ASF culture media and seeded in to a T25 flask. After 4 days, the media was gently aspirated off to remove non-adherent tissue pieces and replenished with fresh ASF culture media. After 7 days post-isolation, the cells were designated as passage 1 and were grown thereafter using a modified 3T3 protocol optimised for efficient ASF maintenance (17). ASFs were routinely cultured in ASF culture media at $37^{\circ} \mathrm{C}$ in a $3 \% \mathrm{O}_{2}$ and $5 \% \mathrm{CO}_{2}$ incubator.

\section{Population doubling measurements}

In the modified 3T3 protocol for ASFs, every third day the cells were trypsinised, counted and then re-seeded at a cell density of $0,8 \times 10^{6}$ cells $/ 10 \mathrm{~cm}$ plate. Cell numbers and viability were calculated using trypan blue exclusion in an automated cell counter, Vi-CELL XR 2.04 (Beckman coulter). Population doubling level (PDL) was calculated as follows: $\mathrm{n}=3.32(\log \mathrm{UCY}-\log \mathrm{I})+\mathrm{X}$, where $\mathrm{n}$ was the final PDL number at the end of a given subculture, UCY was the cell yield at that point, I was the cell number seeded at the start of subculture, and X was the doubling level of the cells used to initiate the subculture being quantified (P-4) (18). In this study, PDL was calculated starting from P4 till P40 and the results are presented as the cumulative population doubling of three separate ASF isolations per genotype.

\section{Growth factor induction}

Induction for the activation kinetics was performed as previously described (19). Briefly, overnight serum starved cells were stimulated using $5 \mathrm{ng} / \mathrm{mL}$ epidermal growth factor (ImmunoTools) for $30 \mathrm{~s}, 2 \mathrm{~min}, 10 \mathrm{~min}$, $30 \mathrm{~min}$, and $60 \mathrm{~min}$ for MEFs, and $2 \mathrm{~min}, 10 \mathrm{~min}$, and $30 \mathrm{~min}$ for ASFs. Cells were harvested and total protein lysates were prepared as previously described (20).

\section{Total protein lysates preparation, pull down and immunoblotting}

Total protein lysates, pull down assay and immunoblotting analysis were performed as described (21). For immunoblotting analysis, the following antibodies were used: anti-actin, anti-ERK1/2, anti-pErk1/2, anti-Akt, anti-pAkt (Ser473), anti-pAkt (Thr308), anti-pEGFR (Tyr1068), anti-pERM, anti-Dusp6, anti-Egfr, anti-phospho-ERM, anti-ERM, anti-Gapdh, all purchased from Cell Signaling. Anti-p21Waf from Santa Cruz Biotechnology.

\section{$R N A$ isolation and $q P C R$ analysis}

Total RNA was isolated using TRIzol (Invitrogen), according to the manufacturer's instructions. For quantitative real time RT-PCR (qPCR), RNA was reverse-transcribed using the High-Capacity cDNA Reverse Transcription Kit (Applied Biosystems) in two steps: step one - initial denaturation of RNA with random primers at $70^{\circ} \mathrm{C}$ for $5 \mathrm{~min}$, followed by the addition of enzymes and reverse transcription at $37^{\circ} \mathrm{C}$ for $120 \mathrm{~min} .20 \mathrm{ng}$ cDNA was amplified and quantified using the iQ SYBR Green Supermix (Bio-Rad), according to the manufacturer's instructions, in the LightCycler ${ }^{\oplus}$ 480 (Roche) under the following conditions: initial denaturation at $95^{\circ} \mathrm{C}$ for $10 \mathrm{~min}$ and 50 cycles of $95^{\circ} \mathrm{C}$ for $15 \mathrm{~s}, 55^{\circ} \mathrm{C}$ for $30 \mathrm{~s}$, and $72^{\circ} \mathrm{C}$ for $30 \mathrm{~s}$. q-PCR was performed in triplicate for each sample. Validated primers from Qiagen for p21WAF (\#QT01752562), p16INK4a (\#QT01164898) and Rpl13a (\#QT00267197) were used. A melting curve analysis was performed for each sample after PCR amplification to verify that the amplicon is homogeneous in the absence of primer dimers and DNA contamination. Cp values were calculated using absolute quantification/ $2^{\text {nd }}$ derivative max (LightCycler ${ }^{\oplus}$ 480 software). The relative expression of each gene is normalized to the expression of their corresponding housekeeping 60S ribosomal protein L13a (Rpl13a). $\triangle \triangle \mathrm{CT}$ were calculated and plots were created using GraphPad Prism 6 software.

\section{ROS measurements (using H2DCFDA)}

For the total cellular ROS assay, $1.5 \times 10^{6}$ cells $/ 10 \mathrm{~cm}$ plate were seeded $\mathrm{O} / \mathrm{N}$ in $\mathrm{ASF}$ culture media and grown in a cell culture incubator at $37^{\circ} \mathrm{C}, 3 \% \mathrm{O}_{2}$ and $5 \% \mathrm{CO}_{2}$. Two hours prior to the H2DCFDA pulse, the cells were trypsinised, pelleted and re-suspended in $1 \mathrm{mLDMEM} / \mathrm{F} 12$ containing only $0.1 \%$ FBS. After recovery, the cells were pulsed with

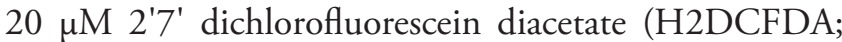
Sigma-Aldrich) for $30 \mathrm{~min}$. Single cells were immediately washed once with ice cold $\mathrm{PBS}^{++}$and analysed using FACs (BD FACSCanto ${ }^{\mathrm{TM}}$ ). A minimum of 3,500 events were recorded in the FITC channel and the median fluorescence for each sample was calculated using Flow $\mathrm{Jo}^{\circledR} \mathrm{V} 10$.

\section{Cell proliferation assay (EdU incorporation)}

Cell proliferation was measured using EdU incorporation followed by detection using click chemistry. For P11, a final concentration of $2 \times 10^{3}$ cells/well were 
cultured overnight in a flat bottomed 96 well plate in a cell culture incubator at $37^{\circ} \mathrm{C}, 5 \% \mathrm{CO}_{2}$ and $3 \% \mathrm{O}_{2}$. FGF was withdrawn 24 hours before pulsing the cells with $10 \mu \mathrm{M}$ EdU for 6 hours. Cells were fixed with 4\% PFA and stained as described below. For P38, $1.5 \times 10^{6}$ cells $/ 10 \mathrm{~cm}$ were cultured overnight, FGF was withdrawn 24 hours before pulsing with $10 \mu \mathrm{M}$ EdU for 3 hours. Single cells were prepared by trypsinisation and fixed with $4 \%$ PFA. EdU was detected using click chemistry with modifications (22). Briefly, fixed cells were washed twice with $\mathrm{PBS}^{++}$, and permeabilised for 10 min with $0.1 \% \operatorname{Triton}^{\mathrm{TM}} \mathrm{X}-100$. The cells were stained with freshly prepared staining solution containing $2 \mathrm{mM} \mathrm{CuSO}_{4}$ (Sigma-Aldrich, from $200 \mathrm{mM}$ stock in $\left.\mathrm{ddH}_{2} \mathrm{O}\right), 10 \mu \mathrm{M}$ Sulfo-Cyanin-3-azid (Lumiprobe, $500 \mu \mathrm{M}$ stock prepared in $\mathrm{ddH}_{2} \mathrm{O}$ ) and $100 \mathrm{mM}$ ascorbic acid (Sigma-Aldrich, freshly prepared in $\mathrm{ddH}_{2} \mathrm{O}$ ) was added in the required amount of $\mathrm{PBS}^{++}$ (Invitrogen), precisely in the above mentioned order. Ascorbate was added at the end, but immediately before EdU staining the cells for $30 \mathrm{~min}$ at RT. After staining, the cells were washed once with $\mathrm{PBS}^{++}$and counterstained with DAPI, followed by further washing. Cells plated in 96 well plates were analysed using a high content screening platform (Cellomics ${ }^{\mathrm{TM}}$, Thermo Scientific). The percentage of EdU-positive cells was calculated from the total number of cells. Single cells were analysed using FACS (BD FACSCanto ${ }^{\mathrm{TM}}$ ), with a minimum of 1000 events recorded in the DAPI and APC channels and the data was analysed using FlowJo ${ }^{\circ}$ V10. The EdU negative population was gated out using no dye control as the reference and the percentage of EdU positive cells over the total events recorded was determined.

\section{Immunofluorescence analysis}

Senescence-associated heterochromatin foci (SAHF) were analysed using HP1 $\alpha$ antibodies (Cell Signaling). Cells were fixed with 4\% PFA for $10 \mathrm{~min}$ at room temperature and permeabilized with $0.25 \%$ Triton X100 in PBS (Sigma). After blocking with 3\% BSA (Sigma, dissolved in $0.25 \%$ Triton $\mathrm{X} 100 / \mathrm{PBS})$ for $1 \mathrm{~h}$ at room temperature, cells were incubated with anti-HP1 $\alpha$ antibody for $1 \mathrm{~h}$ at room temperature. AntiHP1 $\alpha$ detection was performed using anti-rabbit antibody coupled to Alexa 488 (Life Technologies). Cells were mounted using ProLong Gold Antifade with DAPI, for nuclei staining (Life Technologies). Microscopy was performed using Apotome 2 fluorescence microscope (Zeiss).

\section{RESULTS}

\section{H-Ras G12V mutations do not increase MAPK and} Akt activation in mouse embryonic fibroblasts (MEFs)

Various studies using overexpression models have shown that $\mathrm{H}$-Ras oncogenic mutations trigger a constitutive activation of MAPK and Akt, among other Ras pathways.
Here, for H-Ras signalling analysis, we isolated and used MEFs from a CS mouse model. These cells harbour a constitutively active $\mathrm{H}-\mathrm{Ras} \mathrm{G} 12 \mathrm{~V}$ and came from a single $(+/ \mathrm{KI}$, with only one allele carrying the mutated H-Ras G12V) and double knock-in (KI/KI, both alleles were mutated) embryos. Our data confirmed these previous observations (14), that constitutively active H-Ras mutations expressed from their endogenous locus, does not translate into a hyper-activation of the MAPK and Akt pathways (Fig. 1a). In order to elucidate the mechanisms that attenuate oncogenic signalling, we checked the dual specificity phosphatase 6 (Dusp6), a known Erk1/2 activation inhibitory protein (23). Dusp6 expression is regulated by the active Erk1/2 in a feed-forward fashion. To our surprise, immunoblotting analysis showed that only a minor increase in Dusp6 expression was observed in $+/ \mathrm{KI}$ cells, while its expression in $\mathrm{KI} / \mathrm{KI}$ cells was clearly reduced (Fig. 1a, right side panels).

\section{H-Ras G12V mutations induce subtle changes in MAPK and Akt activation kinetics in response to growth factor stimulation}

Pathways associated with Ras GTPases have multiple feedback regulations that can affect targets both upstream and downstream of Ras, regulating the intensity and duration of the signal in response to a stimulus. In fact, previous studies of Ras GTPases mutations have clearly shown that MAPK and Akt activation kinetics are subtle, and that dysregulated activation kinetics analysis can be identified only during stimulation with growth factors (19). Thus, we tested the activation of MAPK and Akt pathways in CS MEFs under epidermal growth factor (EGF) stimulation for various time intervals. Interestingly, activation of MAPK, as measured by phosphorylated Erk1/2 levels, showed subtle differences between wild type and CS MEFs (Fig. 1b, upper and lower panel, respectively). +/KI MEFs showed a stronger activation at early time points (30 s and $2 \mathrm{~min}$ ), but both reached an activation peak after 10 min (Fig. 1b, upper panels). At later time points, MAPK activation in CS cells was reduced when compared to wild type MEFs (30 min). Similar observations were recorded for KI/KI MEFs, with a faster activation at early time points (30 s) (Fig. 1b, lower panels). Next, we studied Akt activation kinetics under the same stimulation conditions. In CS cells, Akt activation pattern was similar to MAPK, an early activation in both, $+/ \mathrm{KI}$ and KI/KI CS MEFs (30 s and $2 \mathrm{~min}$ ), while at later time points $(30 \mathrm{~min}$ and $60 \mathrm{~min})$, Akt activation was stronger in wild type cells (Fig. 1b, upper and lower panels). To sum it all up, although MAPK and Akt activation in CS and wild type cells reached a maximum after 10 min of EGF induction, the mutated cells showed an earlier activation in reaching the stimulation peak by $10 \mathrm{~min}$, and at the same time, their inactivation was faster than that in the wild type cells. 


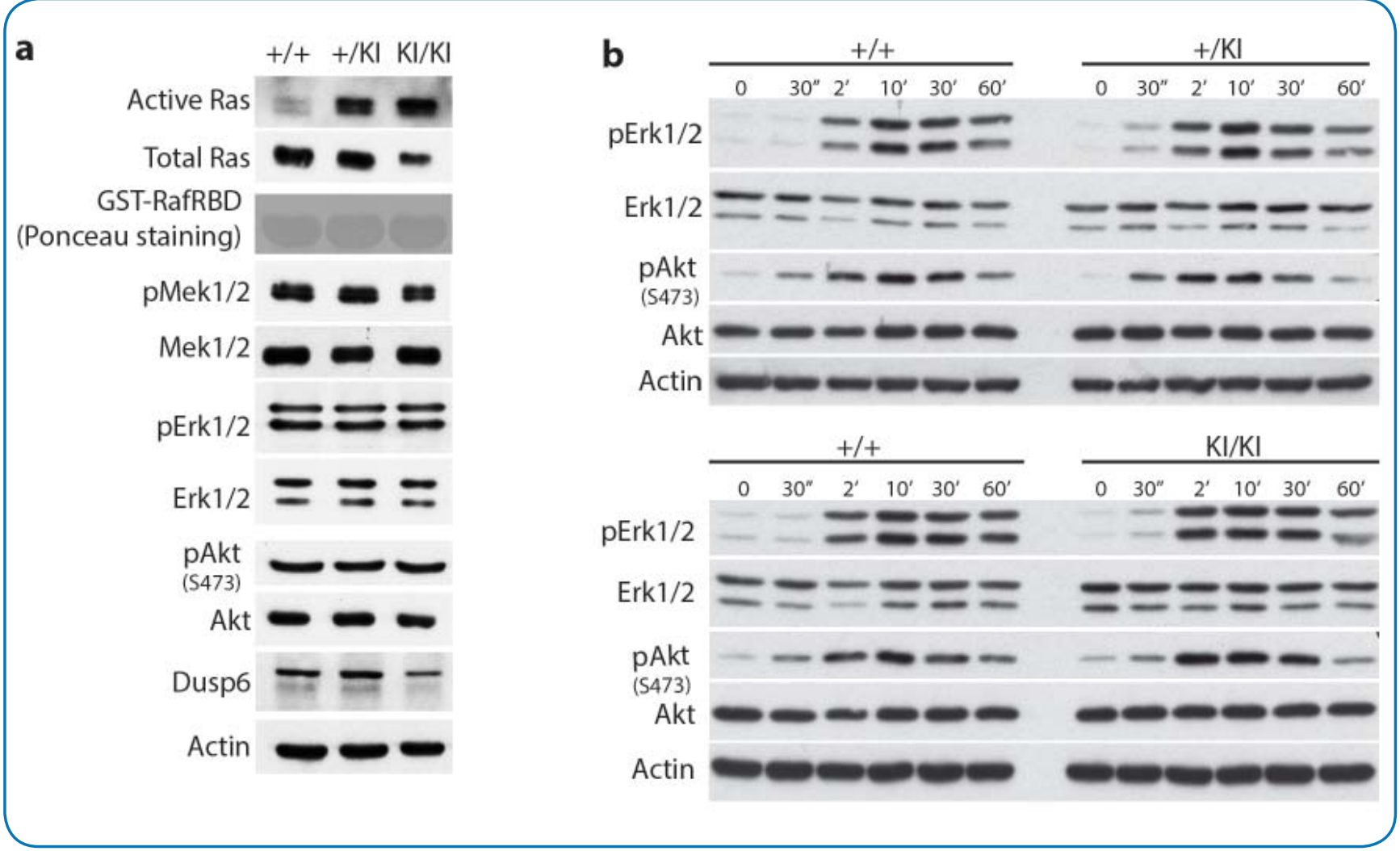

Fig. 1. - MAPK and Akt signalling in CS mouse embryonic fibroblasts (MEFs). Representative immunoblotting analysis of cleared cell lysates using specific antibodies against total and phospho-proteins: Ras, phospho-Mek1/2 (Thr217/Thr221), Mek1/2, phospho-Erk1/2 (Thr202/Tyr204), Erk1/2, Dusp6 and actin. Ponceau staining of GST-Raf RBD shows similar levels of bait used for pull down assay. Actin was used as control for protein normalization. a) Signalling downstream of H-Ras in mouse embryonic fibroblasts (MEFs). Pull down assay shows an increase of active H-Ras levels in CS MEFs, an increase which was not translated into a hyper-activation of MAPK and Akt pathways (left side panels). Molecular analysis of the Erk1/2 inhibitory protein Dusp6 shows similar levels to wild type cells. b) EGF-induced ERK1/2 and Akt activation kinetics in MEFs. MEFs were stimulated using $5 \mathrm{ng} / \mathrm{mL}$ EGF and harvested at the specified time points. Immunoblotting analysis revealed subtle changes in MAPK and Akt activation kinetics in CS cells, an early activation for CS MEFs (after 30 sec and 2 min stimulation) and a faster inactivation after $30 \mathrm{~min}$, when compared to wild type cells.

Basal activation of H-Ras G12V stimulates the Akt pathway in mouse adult skin fibroblasts (ASFs), whereas EGF induction induces alterations in MAPK and Akt activation kinetics

To expand on our analysis of the dysregulated H-Ras signalling to adult primary cells, we isolated ASFs and used them to study cellular signalling. We first examined MAPK and Akt activation in ASFs in the absence of any stimulation at passage 7 (P7). Immunoblotting analysis showed that despite a minor increase in Mek1/2 activation, Erk1/2 activity levels did not change in the CS cells when compared to wild type. Similar to MEFs, Dusp6 expression was not increased by the presence of H-Ras G12V mutations, and the expression levels were comparable in CS and wild type ASFs (Fig. 2a). We tested the activation of Akt pathways in CS ASFs, using two phospho-Akt antibodies, one recognising a Pdk1-mediated activating phosphorylation (Thr308) and the another recognising a mammalian target of rapamycin complex 2 (mTorc2)-mediated phosphorylation (Ser473). Surprisingly, immunoblotting analysis identified that as a result of $\mathrm{H}$-Ras hyper-activation, both activating phosphorylations were increased in CS ASFs (Fig. 2a), in contrast to our own observations in MEFs. Similar to our analysis in MEFs, we tested whether EGF induction reveals subtle changes during MAPK and Akt activation. EGF induction showed similar activation kinetics for MAPK measured by Erk1/2 phosphorylation in both wild type and CS ASFs (Fig. 2b and Supplementary Fig. 1). In contrast to this observation, an evident activation of Raf1 kinase in response to constitutive active $\mathrm{H}$-Ras and in the absence of stimulation was not subsequently translated into Mek1/2 and Erk1/2 activation. On the other hand, we identified that CS cells showed an increased Akt activation in the absence of stimulation (time 0) for both Akt activating phosphorylation sites, while the activation at subsequent time points ( 2 and $5 \mathrm{~min}$ ) was similar to the wild type cells (Fig. 2d and Supplementary Fig. 1). In CS cells, Akt activation in response to EGF remained higher than in the wild type cells after $30 \mathrm{~min}$ of stimulation, further confirming that H-Ras mutations induced subtle changes in pathway activation kinetics. 


\section{H-Ras G12V alters the EGF-mediated activation of} Egfr and ERM in ASFs

EGF induction analysis in ASFs showed subtle changes in the activation kinetics downstream of H-Ras. Next, we focused on Egfr stimulation, a MAPK component located upstream of Ras. EGF binds to Egfr, which in turn induces autophosphorylation of the receptor and the recruitment and stimulation of upstream activators of Ras GTPases. EGF-mediated activation kinetics of Egfr were measured by levels of phosphorylated Egfr at Tyr1068, and we observed that in contrast to CS MEFs (Supplementary Fig. 2), the phosphorylation of Egfr was reduced in mutated ASFs (Fig. 2c). To our surprise, the reduced activation is related to a strong reduction of Egfr expression in mutated cells. We then tested the activation of ERM (Ezrin, Radixin, Moesin) proteins, which are known to associate with Egfr (24), and to sustain Ras activation (25). Phosphorylation of Ezrin at Thr567 (Radixin Thr564, Moesin Thr558) in response to growth factor stimulation induces the activation of ERM proteins (26). In mutant ASFs ( $+/ \mathrm{KI}$ and $\mathrm{KI} / \mathrm{KI})$, we observed a strong phosphorylation of ERM proteins under basal conditions (time 0 ), while in ${ }^{+} / \mathrm{KI}$ cells, the activation was reduced after stimulation (Fig. 2c). At later time points (time 10 and $30 \mathrm{~min}$ ), ERM activation in KI/KI cells was higher than in wild type and +/KI cells.

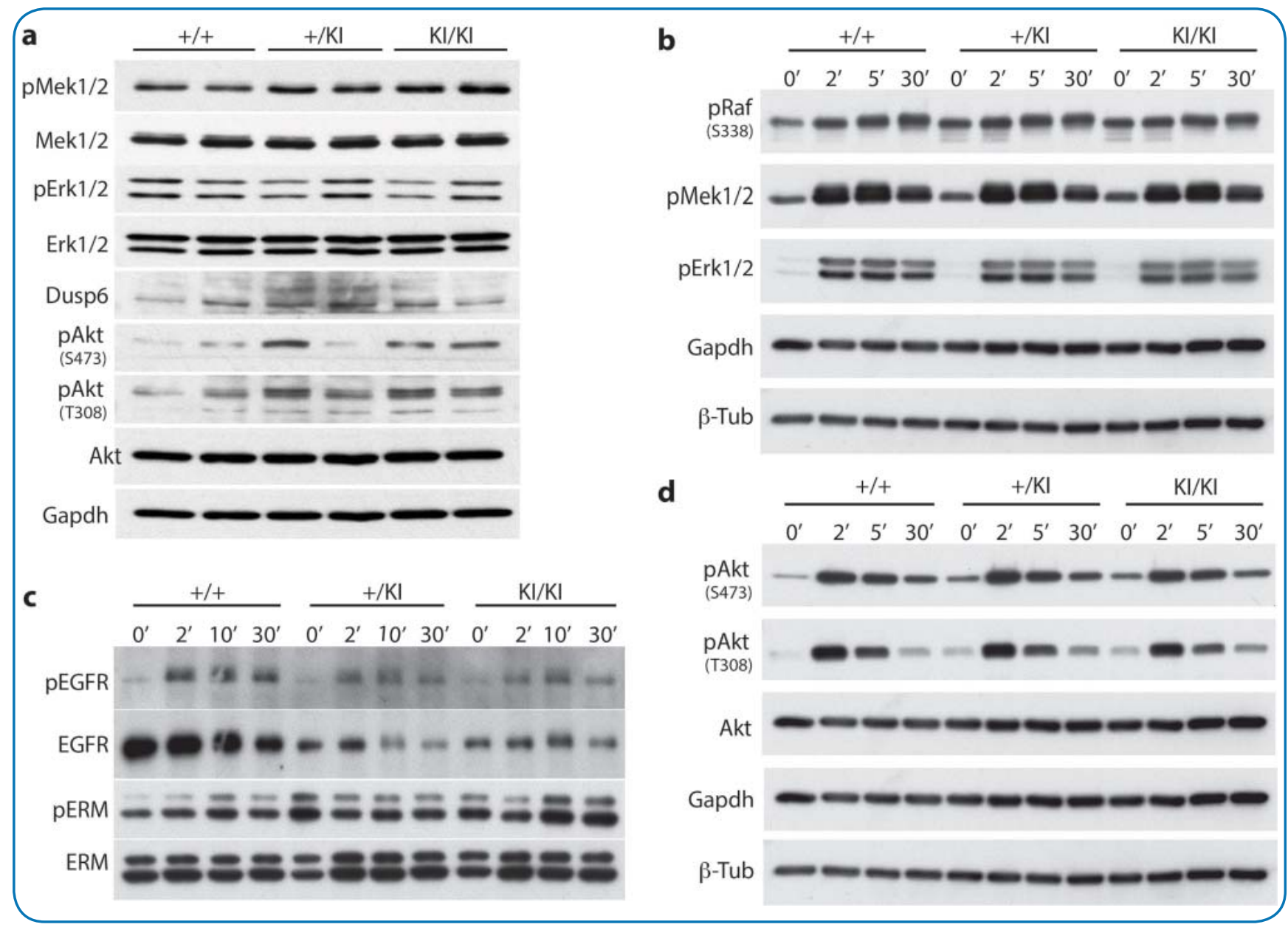

Fig. 2. - MAPK and Akt signalling in CS adult mouse skin fibroblasts (ASFs). Representative immunoblotting analysis of cleared cell lysates was performed using specific antibodies against total and phospho-proteins. a) Signalling downstream of $H$-Ras in mouse adult skin fibroblasts (ASFs) in the absence of stimulation (P7). MAPK activation does not change in CS cells and its levels are similar to wild type cells. In contrast to MAPK, H-Ras mutations activate Akt, confirmed by the increased levels of phosphorylated Akt (Thr308 and Ser473). Similar to MEFs analysis, Dusp6 expression in ASFs does not change in response to H-Ras G12V mutations. Gapdh was used as the control for protein normalization. $b$ ) EGF-induced activation of Egfr and ERM protein in ASFs. Activation of Egfr is reduced in mutant ASFs and probably causes a reduced expression of Egfr. ERM proteins activation under basal conditions (time 0) was increased in mutant ASFs, as well as under EGF stimulation. In contrast to basal activation in $+/$ KI cells, the activation of ERM was reduced under EGF stimulation, while $\mathrm{KI} / \mathrm{KI}$ cells show a continuous activation, even at later time points (time 10 and $30 \mathrm{~min}$ ). c) EGF-induced MAPK activation kinetics in CS ASFs. MAPK analysis has shown no difference in Erk1/2 activation between CS and wild type cells. d) EGF-induced Akt activation in CS ASFs. CS mutations induce Akt activation in the absence of stimulation, as well as a prolonged signalling ( $30 \mathrm{~min}$ ), demonstrated by the increased phospho-Akt levels at time 0 and $30 \mathrm{~min}$. Gapdh and $\beta$-tubulin were used as the controls for protein normalization. 


\section{H-Ras G12V induces a weak oncogene-induced senescence-like phenotype in ASFs}

Given that CS ASFs have an increased basal mitogenic signalling, we wanted to explore the cellular outcome of such a dysregulated Ras signalling. Unlike human primary cells, mouse primary fibroblasts have longer telomeres, allowing for longer culturing times, or until they theoretically attain replicative senescence due to telomere attrition (27). On the other hand, many studies have shown that mouse primary fibroblasts will stop proliferation and will ultimately undergo senescence in response to oncogenic Ras overexpression (28). Here, we report that in contrast to wild type ASFs, cumulative population doubling in CS cells was steadily reduced during our observation over a period of more than 30 cell passages (Fig. 3a). To conclusively prove that cellular proliferation was indeed reduced, we performed EdU incorporation in CS cells and we observed that in contrast to P7, these cells showed a gene-dosage dependent reduction of proliferation when measured at P38 (Fig. 3b). Same, the absolute cell number at late passage was clearly reduced in CS ASFs (Supplementary Fig. 4). Next, we tried to identify the molecular targets responsible for this reduced proliferation and the possible induction of oncogene-induced senescence. It has long been known that $p 16 \operatorname{Ink} 4 a$ and $p 21 \mathrm{Waf}$ induce cell cycle arrest in response to DNA damage and mitotic stress induced by oncogenes (29). We set out to determine the expression of these two targets at an early passage (P3) and a late passage (P25). In contrast to wild type cells, CS ASFs showed a constant increase in both p16ink4a and p21waf, which correlates with the reduction in cumulative population doubling (Fig. 3c). Additionally, p21Waf immunoblotting conclusively proved its upregulation at a late passage (Fig. 3c, inset). Though we tested numerous antibodies, we could not detect $p 16$ Ink $4 a$ protein expression, due to their poor quality. Another hallmark of OIS is the increase in ROS production, which in turn accelerates the senescence phenotype. In normal conditions, ROS are used as signalling molecules and are controlled by the Ras/PI3K/Rac1/NADPH oxidases pathway. Under oncogenic Ras stress, the production of ROS increases and results in sustained cellular damage. Because we observed the induction of OIS markers, we tested in a late passage (P36) the production of ROS via 2',7'-dichlorodihydrofluorescein diacetate ( $\left.\mathrm{H}_{2} \mathrm{DCFDA}\right)$ incorporation and FACS analysis. Our analysis shows an increase in ROS production in CS ASFs irrespective to the cellular genotype (Fig. 3d). Furthermore, we inspected the induction of senescence-associated heterochromatin foci (SAHF) and we used as marker HP1 $\alpha$, known marker induced during cellular senescence (30-32). Microscopy analysis clearly shows that in contrast to a uniform distribution in wild type ASFs, CS cells induce HP1 $1 \alpha$-positive foci (Fig. 3e and Supplementary Fig. 3).
Unfortunately, SA- $\beta G$ al could not be used as a senescence marker because the knock-in H-Ras G12V contains a $\beta g a l$ reporter gene to monitor H-Ras G12V tissue-specific expression. Additional markers (e.g., DNA damage marker $\gamma \mathrm{H} 2 \mathrm{AX}$ ) were tested, but we could not detect clear differences between wild type and CS ASFs (data not shown). In our model, the expression of oncogenic $\mathrm{H}$-Ras is physiological and we presume that in contrast to the overexpression of oncogenes, the OIS-like phenotype occurs at a slower pace.

\section{DISCUSSION}

CS is a developmental disorder arising from oncogenic H-Ras G12V germline mutations. Despite harbouring oncogenic mutations, the cancer incidence is reduced, implying that protective mechanisms are triggered in both patients and the CS mouse model. Our molecular analysis in embryo and adult fibroblasts showed that the MAPK and Akt pathways are not upregulated, although subtle changes in their activation were identified. MEFs are primary cells extensively studied for the effects of oncogenes overexpression on cellular proliferation and tumour initiation (28). In contrast to somatic mutations, our data using primary fibroblasts expressing H-Ras G12V from its endogenous locus, did not show hyperactivation of MAPK in both primary cells types. Only a strong activation of the Akt pathway occurred in serum-starved CS ASFs, and we believe that, in this cellular model, activated Akt may well be responsible for the dysregulated cellular signalling. This observation prompts us to assume that germline H-Ras G12V mutations are tolerated during embryonic development because MAPK and Akt pathways are kept at normal levels, through unknown mechanisms. Furthermore, we identified subtle alterations of MAPK and Akt pathways when cells were stimulated with EGF. Subtle changes in the activation kinetic of EGF-induced MAPK signalling were previously described in an in vitro study in cells expressing Noonan syndrome-associated K-Ras mutants or in CS patients' fibroblasts (19, 33). In our molecular analysis of MEFs and ASFs, we could not associate Dusp6, a classical negative regulator of MAPK, with the reduced MAPK activation in cells harbouring H-Ras G12V mutations. Because the MAPK phosphatases family comprises other members which can also affect the classical MAPK or stress-induced MAPK, we cannot exclude the involvement of another MAPK inhibitory phosphatase (e.g., DUSP1). In addition to the MAPK signalling intensity, subcellular localisation of its components has an influence on the outcome of cellular signalling. Another intriguing observation is that Raf kinase activation was increased in CS ASFs in the absence of EGF stimulation (Fig. 2b). Though Raf kinases activation is canonically correlated with MAPK signalling, few studies proved that Raf involved in the 
a
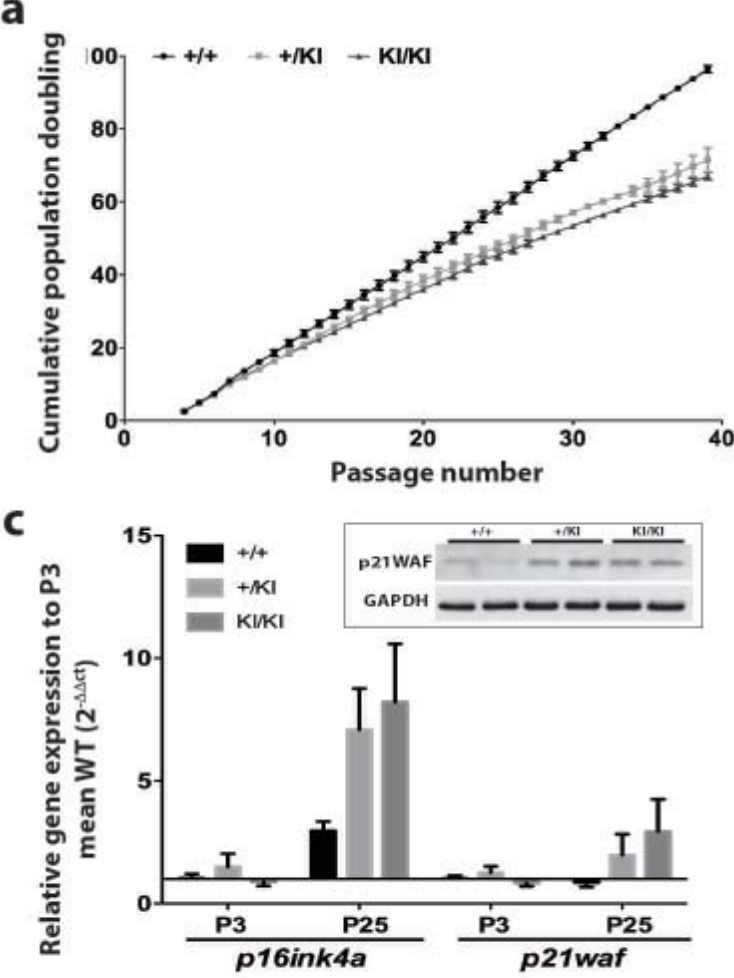

e

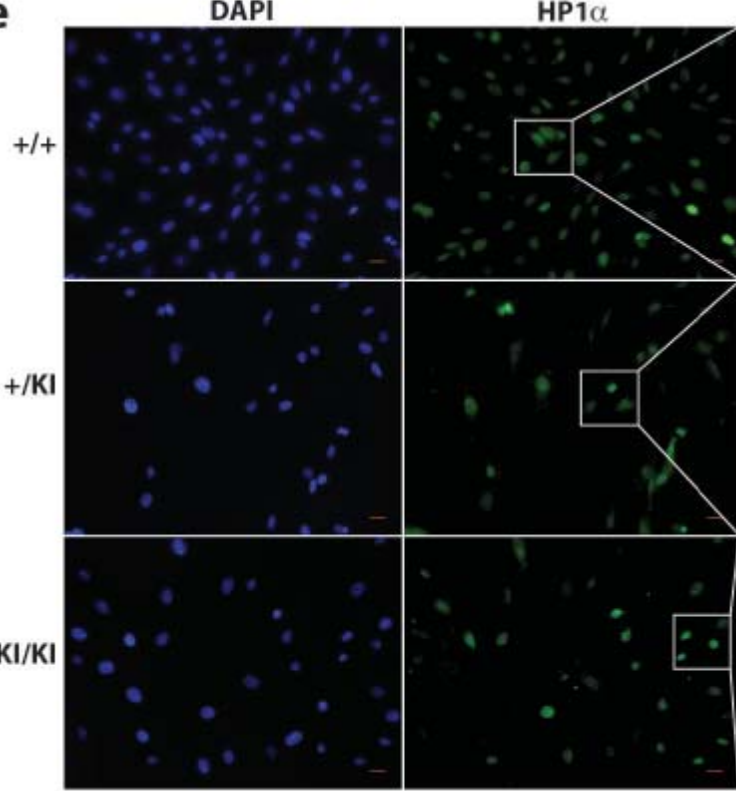

b
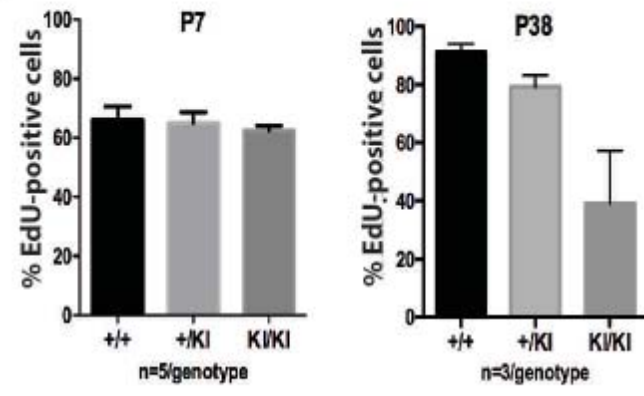

d

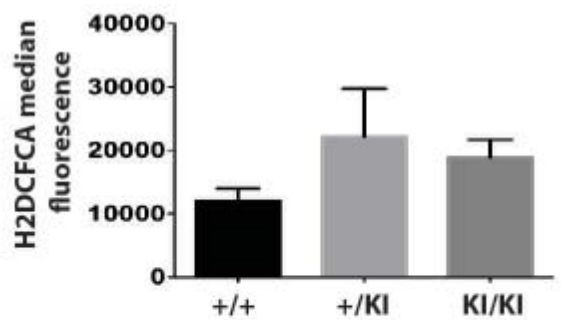

HP1 $\alpha$ (magnified view)

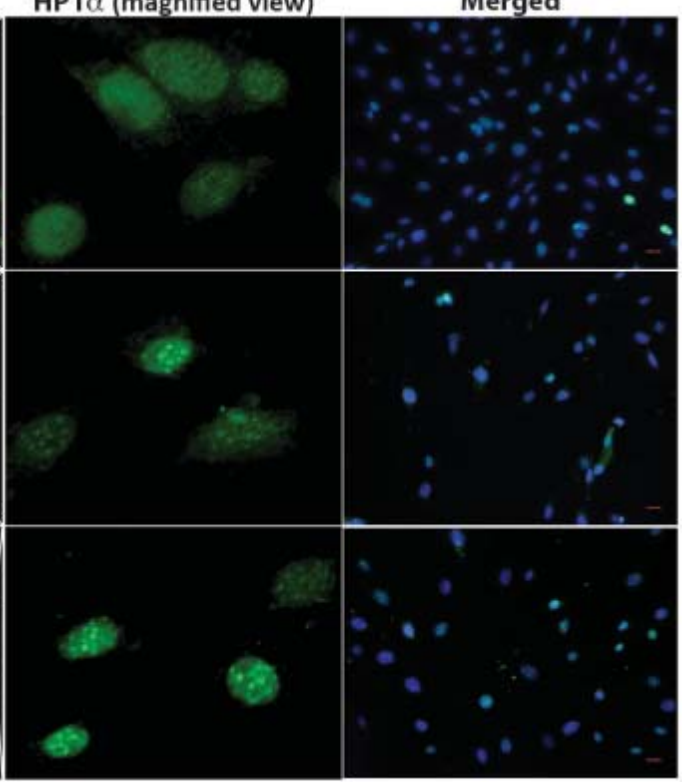

Fig. 3. - CS mutations in ASFs reduce cellular proliferation and activate an oncogene-induced senescence-like phenotype. a) CS ASFs display a reduced cumulative population doubling. ASFs were cultured for 40 passages and cumulative population doubling was calculated as described in the methods section for 3 independent cell lines per genotype; $b$ ) H-Ras mutations reduce cellular proliferation in CS cells. Cellular proliferation measured using EdU incorporation assay shows reduced proliferation in CS cells. For passage 7 and passage 32, cells were pulsed with $10 \mu \mathrm{M}$ EdU for 6 hours, fixed, stained and analysed using a high content screening platform (Cellomics ${ }^{\mathrm{TM}}$, Thermo Scientific) (P7) or FACS Canto (P38); c) H-Ras G12V induces the expression of p16ink $4 a$ and 21 waf cell cycle inhibitory proteins in CS cells. The expression of cell cycle inhibitory proteins was performed using qPCR analysis (p16ink4a,p21waf) and immunoblotting (p21Waf and Gapdh as control); d) CS mutations induce ROS production. Intracellular ROS production was measured via FACS analysis of cells incorporating 2'7'-dichlorodihydrofluorescein diacetate (H2DCFDA). Measurements quantification was performed using FlowJo.

e) CS ASFs senescence-associated heterochromatin foci (SAHF). Representative images of immunofluorescence analysis of SAHF, detected using HP1 a antibodies (green). In contrast to wild type cells, HP1a localizes in distinct foci ( $3^{\text {rd }}$ panels). Images were acquired using Apotome 2 fluorescence microscope (Zeiss). Nuclei were detected using 4',6-diamidino-2-phenylindole (DAPI, blue). Scale bar $20 \mu \mathrm{m}$. For statistical analysis, all values were plotted using GraphPad Prism 6 software and are represented as \pm SEM 
phosphorylation of eukaryotic translation elongation factor 1A, apoptosis-linked gene-2, Bcl-2-associated death promoter and their phosphorylation by Raf kinase appear to induce prosurvival functions in cells (34-36) and ultimately to the dysregulation of cellular homeostasis. In addition to changes in MAPK and Akt activation downstream of Ras, we identified significant changes in the expression of Egfr and the activation of ERM proteins. The reduction of Egfr activation was directly linked to the reduction in its expression, and in CS cells may well be the trigger for a reduced MAPK activation downstream of Ras. From pancreatic KRAS cancer models, it is known that oncogenic K-Ras activity is dependent on Egfr signalling (37), so we can hypothesize that the reduction of Egfr expression by a currently elusive mechanism may conceivably be a protective mechanism against tumorigenic transformation of CS fibroblasts. Analysing the cellular signalling upstream of $\mathrm{H}$-Ras, we identified an increased activation of ERM proteins in the absence of growth factor stimulation. ERM are protein linkers between the membrane receptors and the cortical cytoskeleton, in turn modulating receptor output. Their expression levels were reportedly increased in various tumours (25). A direct link between ERM proteins and Ras activation was previously reported (38); therefore, we were not surprised to observe an increased ERM activation in the CS cells expressing oncogenic H-Ras. However, the role of ERM is not limited only to Ras activation, as ERM interactions can modulate receptor abundance, localisation and via interacting with other proteins, can influence cytoskeletal signalling, cellular polarity, mitosis, among many other processes (39). Ezrin phosphorylation plays an essential role in insulin secretion from the pancreas, and for future studies it will be essential to understand whether there is a link between ERM activation and hyperinsulinemia identified in CS patients (40). We hypothesize that signalling alterations upstream and downstream of H-Ras lack the potency to induce cancer, but have a detrimental effect on the cellular and tissues homeostasis during embryonic development and throughout the life span of CS patients.

Numerous studies have shown that oncogenic mutations affecting Ras GTPase promote tumorigenic transformation, but at the same time, during the early steps of tumour initiation, they activate OIS. OIS is a protective cellular mechanism against uncontrolled proliferation and it is characterised by a permanent cell cycle arrest, increased production of reactive oxygens species (ROS), activation of the DNA damage response and a specific secretory phenotype (42-44). In vitro overexpression studies of oncogenic Ras in human primary fibroblasts has revealed that the induction of oncogene-induced senescence (45) is characterised by the activation of senescence-associated beta-galactosidase (SA- $\beta \mathrm{Gal})$ and a strong expression of p16Ink4a. We observed in CS ASFs the induction of a OIS-like phenotype, characterized by the reduction of cellular proliferation, the expression of cell cycle inhibitory genes p16ink4a and p21waf expression increases towards late passages.

Furthermore, we have identified the induction of HP1 $\alpha$-positive senescence-associated heterochromatin foci (SAHF), but, to our surprise, there were no differences in $\gamma \mathrm{H} 2 \mathrm{AX}$-positive DNA damage foci. Moreover, an increased ROS production was noticed in late passages. In contrast to overexpression cellular models, our cells carry $H$-Ras oncogene (single or both alleles mutated) expressed at physiological levels and we presume that the initiation and establishment of OIS-like phenotype in these cells occurs at a slower pace. Nevertheless, the stronger ROS production in CS cells will induce further DNA and cellular damage, and thus ultimately drive the cells into senescence and a complete cell cycle arrest. One important aspect in senescence is that in contrast to humans, mice have longer telomeres, therefore until they reach a critical telomere length and trigger a strong senescence response, a spontaneous immortalization may occur (46). Moreover, the strength of an oncogene in mouse models has a strong impact over the balance between cellular proliferation and senescence. Previous work showed that oncogenic K-Ras (more potent oncogene than H-Ras) in mouse primary fibroblasts does not induce senescence, rather accelerates proliferation and triggers immortalization after only few days in culture (47). In our cellular model, a less potent $\mathrm{H}$-Ras oncogene slows down proliferation and induces a senescence-like phenotype, but the spontaneous immortalization in mouse cells cannot be excluded at later passage number.

Based on these in vitro observations, we hypothesize that within an organism, in vivo context, the complexity of cellular types and the influence of various secreted factors produced by the niche could ultimately stimulate OIS in highly proliferative cells like the stem cells (43). Therefore, future studies in the CS mouse model could unravel whether systemic changes will drive proliferating cells into senescence. Because the patients display an age-dependent worsening phenotype, we cannot exclude that senescence is a possible contributor to the CS pathologies. In an organism, the effect of OIS is not limited only to proliferative cells, but can also influence terminally differentiated cells, like hepatocytes (48). Induction of senescence in hepatocytes could induce changes in metabolic processes that can be responsible for CS pathologies. In future studies using CS animal models, we intend to expand the analysis to organs and tissues and understand the systemic changes induced by OIS and its correlation to CS pathologies.

Taking into account our experimental data, we think that the induction of the senescence-like phenotype in CS primary fibroblasts may well be responsible for blocking the tumorigenic transformation. At the 
same time, cumulative effects of dysregulated pathways upstream and downstream of H-Ras, and the induction of oncogene-induced senescence-like phenotype may be responsible for the induction of CS pathologies.

Author contributions: S.C. and T.S. performed the experiments, S.C. reviewed the manuscript, ICC designed the experiments and wrote the manuscript. Acknowledgments: We thank Dr. Steven John Smith for critical reading of the manuscript and

\section{REFERENCES}

1. Kratz CP, Niemeyer CM, Zenker M, An unexpected new role of mutant Ras: perturbation of human embryonic development, J. Mol. Med. (Berl)., 85, 227-235 (2007).

2. Aoki Y, Niihori T, Kawame H, Kurosawa K, Ohashi H, Tanaka Y, et al, Germline mutations in HRAS proto-oncogene cause Costello syndrome, Nat. Genet., 37, 1038-1040 (2005).

3. White SM, Graham JM, Kerr B, Gripp K, Weksberg R, Cytrynbaum C, et al, Adult phenotype in Costello syndrome, Am.J. Med. Genet., 136A, 128-135 (2005).

4. Kratz CP, Franke L, Peters H, Kohlschmidt N, Kazmierczak B, Finckh U, et al., Cancer spectrum and frequency among children with Noonan, Costello, and cardio-facio-cutaneous syndromes, Br. J. Cancer, 112, 1392-1397 (2015).

5. Karnoub AE, Weinberg RA, Ras oncogenes: split personalities, Nat. Rev. Mol. Cell. Biol., 9, 517-531 (2008).

6. Malumbres M, Barbacid M. RAS oncogenes: the first 30 years, Nat. Rev. Cancer, 3, 459-465 (2003).

7. Wittinghofer A, Vetter IR. Structure-Function Relationships of the G Domain, a Canonical Switch Motif, Annu. Rev. Biochem., 80, 943-971 (2011).

8. Ahmadian MR, Stege P, Scheffzek K, Wittinghofer A, Confirmation of the arginine-finger hypothesis for the GAP-stimulated GTP-hydrolysis reaction of Ras, Nat. Struct. Biol., 4, 686-689 (1997).

9. Scheffzek K, Ahmadian MR, Kabsch W, Wiesmuller L, Lautwein A, Schmitz F, et al., The Ras-RasGAP complex: Structural basis for GTPase activation and its loss in oncogenic Ras mutants, Science, 277, 333-338 (1997).

10. Estep AL, Tidyman WE, Teitell MA, Cotter PD, Rauen KA. HRAS mutations in Costello syndrome: Detection of constitutional activating mutations in codon 12 and 13 and loss of wild-type allele in malignancy, Am. J. Med. Genet. Part A, 140A, 8-16 (2004).

11. Lorenz S, Petersen C, Kordaß U, Seidel H, Zenker M, Kutsche K. Two cases with severe lethal course of Costello syndrome associated with HRAS p.G12C and p.G12D, Eur. J. Med. Genet., 55, 615-619 (2012).

12. Piccione M, Piro E, Pomponi MG, Matina F, Pietrobono R, Candela $\mathrm{E}$, et al., A premature infant with Costello syndrome due to a rare $G 13 C$ HRAS mutation,

Am. J. Med. Genet. Part A, 149A, 487-489 (2009).

13. Viosca J, Schuhmacher AJ, Guerra C, Barco A, Germline expression of H-Ras $(G 12 \mathrm{~V})$ causes neurological deficits associated to Costello syndrome, Genes Brain Behav., 8, 60-71 (2009).

14. Schuhmacher AJ, Guerra C, Sauzeau V, Canamero M, Bustelo X, Barbacid M, A mouse model for Costello syndrome reveals an Ang II-mediated hypertensive condition, J. Clin. Invest., 118, 2169-2179 (2008).

15. Goodwin AF, Tidyman WE, Jheon AH, Sharir A, Zheng X, Charles C, et al., Abnormal ras signaling in costello syndrome (CS) negatively regulates enamel formation, Hum. Mol. Genet., 23, 682-692 (2014).

16. Paquin A, Hordo C, Kaplan DR, Miller FD, Costello syndrome
Prof. Mariano Barbacid (CNIO, Spain) for the CS mouse model. The work was supported by: Leibniz Graduate School on Aging to S.C.; Career Development Fellowship of the Leibniz Institute on Aging to I.C.C.; Bundesministerium für Bildung und Forschung/German Network for Rasopathy Research-GeNeRARe, grant number 01GM1519F to I.C.C. Conflicts of interest: None to declare.

H-Rasalleles regulate cortical development, Dev. Biol., 330, 440-451 (2009).

17. Todaro GJ, Green H, Quantitative studies of the growth of mouse embryo fibroblasts in culture and thier development into established lines, J. Cell. Biol., 17, 299 LP-313 (1963).

18. Hayflick L, Tissue Culture Methods and Applications. Kruse PF and Patterson MK, eds, Academic Press, New York, 1973, 220 pp.

19. Cirstea IC, Gremer L, Dvorsky R, Zhang S-C, Piekorz RP, Zenker M, et al., Diverging gain-of-function mechanisms of two novel KRAS mutations associated with Noonan and cardio-faciocutaneous syndromes, Hum. Mol. Genet., 22, 262-270 (2013).

20. Gremer L, Merbitz-Zahradnik T, Dvorsky R, Cirstea IC, Kratz CP, Zenker M, et al., Germline KRAS Mutations Cause Aberrant Biochemical and Physical Properties Leading to Developmental Disorders, Hum. Mutat., 32, 33-43 (2011).

21. Cirstea IC, Kutsche K, Dvorsky R, Gremer L, Carta C, Horn D, et al., A restricted spectrum of NRAS mutations causes Noonan syndrome, Nat. Genet. 42, 27-29 (2010).

22. Salic A, Mitchison TJ, A chemical method for fast and sensitive detection of DNA synthesis in vivo, Proc. Natl. Acad. Sci., 105, 2415-2420 (2008).

23. Maillet M, Purcell NH, Sargent MA, York AJ, Bueno OF, Molkentin JD, DUSP6 (MKP3) Null Mice Show Enhanced ERK1/2 Phosphorylation at Baseline and Increased Myocyte Proliferation in the Heart Affecting Disease Susceptibility, J. Biol. Chem., 283, 31246-31255 (2008).

24. Krieg J, Hunter T, Identification of the two major epidermal growth factor-induced tyrosine phosphorylation sites in the microvillar core protein ezrin, J. Biol. Chem., 267, 19258-19265 (1992).

25. Clucas J, Valderrama F, ERM proteins in cancer progression, J. Cell Sci., 127, 267 LP-275 (2014).

26. Gautreau A, Louvard D, Arpin M, Morphogenic Effects of Ezrin Require a Phosphorylation-Induced Transition from Oligomers to Monomers at the Plasma Membrane, J. Cell. Biol., 150, 193 LP-204 (2000).

27. Gomes NM V, Ryder OA, Houck ML, Charter SJ, Walker W, Forsyth NR, et al., Comparative biology of mammalian telomeres: hypotheses on ancestral states and the roles of telomeres in longevity determination, Aging Cell, 10, 761-768 (2011).

28. Serrano M, Lin AW, McCurrach ME, Beach D, Lowe SW, Oncogenic ras Provokes Premature Cell Senescence Associated with Accumulation of p53 and p16INK4a, Cell, 88, 593-602 (1997).

29. Yaswen P, Campisi J, Oncogene-Induced Senescence Pathways Weave an Intricate Tapestry, Cell, 128, 233-234 (2007).

30. Collado M, Serrano M, Senescence in tumours: evidence from mice and humans, Nat. Rev. Cancer, 1, 51-57 (2010).

31. Aird KM, Zhang R, Detection of Senescence-Associated Heterochromatin Foci (SAHF), In: Cell Senescence: Methods and Protocols, Galluzzi L, Vitale I, Kepp O, Kroemer G, eds, NJ: Humana Press, 185-196 (2013). 
32. Liu J, Yin X, Liu B, Zheng H, Zhou G, Gong L, et al., HP1 $\alpha$ mediates defective heterochromatin repair and accelerates senescence in Zmpste24-deficient cells, Cell Cycle, 8, 1237-1247 (2014).

33. Rosenberger G, Meien S, Kutsche K, Oncogenic HRAS Mutations Cause Prolonged PI3K Signaling in Response to Epidermal Growth Factor in Fibroblasts of Patients With Costello Syndrome, Hum. Mutat., 30, 352-362 (2009).

34. Chen C, Sytkowski AJ, Apoptosis-linked gene-2 connects the Raf-1 and ASK1 signalings, Biochem. Biophys. Res. Commun., 1, 51-57 (2005).

35. Ye DZ, Jin S, Zhuo Y, Field J, p21-Activated Kinase 1 (Pak1) Phosphorylates BAD Directly at Serine 111 In Vitro and Indirectly through Raf-1 at Serine 112, PLoS One, 11, e27637 (2011).

36. Sanges C, Scheuermann C, Zahedi RP, Sickmann A, Lamberti A, Migliaccio N, et al., Raf kinases mediate the phosphorylation of eukaryotic translation elongation factor $1 \mathrm{~A}$ and regulate its stability in eukaryotic cells, Cell Death Dis., 3:e276 (2012).

37. Navas C, Hernández-Porras I, Schuhmacher AJ, Sibilia M, Guerra C, Barbacid M, EGF receptor signaling is essential for $K$-Ras oncogene-driven pancreatic ductal adenocarcinoma, Cancer Cell, 22, 318-330 (2012).

38. Sperka T, Geißler KJ, Merkel U, Scholl I, Rubio I, Herrlich P, et al., Activation of Ras Requires the ERM-Dependent Link of Actin to the Plasma Membrane, PLoS One, 6, e27511 (2011).

39. Fehon RG, McClatchey AI, Bretscher A, Organizing the cell cortex: the role of ERM proteins, Nat. Rev. Mol. Cell Biol., 11, 276-287 (2010).

40. Lopez JP, Turner JR, Philipson LH, Glucose-induced ERM protein

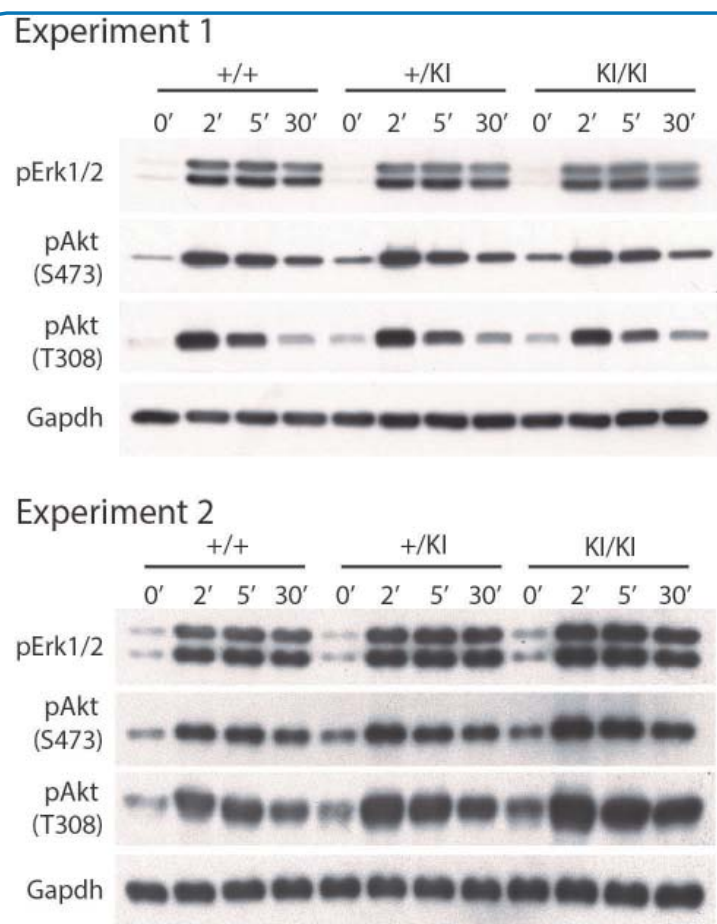

Experiment 3

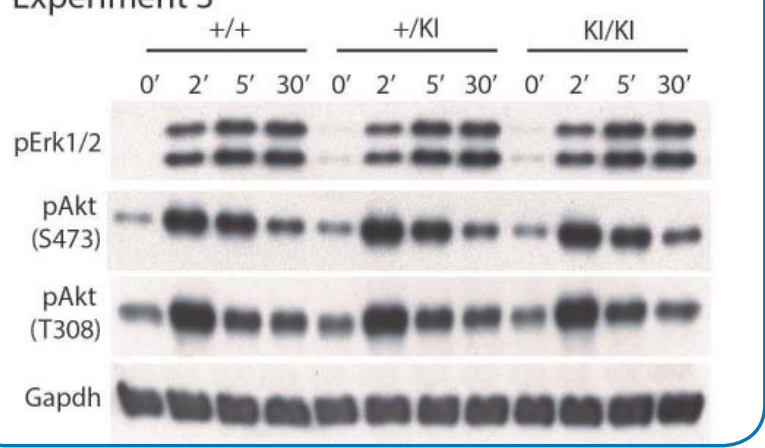

activation and translocation regulates insulin secretion,

Am. J. Physiol. - Endocrinol. Metab., 299, E772-E785 (2010).

41. Rodríguez J, Crespo P, Working Without Kinase Activity: Phosphotransfer-Independent Functions of Extracellular Signal-Regulated Kinases, Sci. Signal., 4, re3 LP-re3 (2011).

42. Campisi J, d'Adda di Fagagna F, Cellular senescence: when bad things happen to good cells, Nat. Rev. Mol. Cell. Biol., 8, 729-740 (2007).

43. Tchkonia T, Zhu Y, van Deursen J, Campisi J, Kirkland JL, Cellular senescence and the senescent secretory phenotype: therapeutic opportunities, J. Clin. Invest., 123, 966-972 (2013).

44. Takahashi A, Ohtani N, Yamakoshi K, Iida S, Tahara H, Nakayama K, et al., Mitogenic signalling and the p16INK4a-Rb pathway cooperate to enforce irreversible cellular senescence, Nat. Cell Biol., 8, 1291-1297 (2006).

45. Niihori T, Aoki Y, Okamoto N, Kurosawa K, Ohashi H, Mizuno $\mathrm{S}$, et al., HRAS mutants identified in Costello syndrome patients can induce cellular senescence: possible implications for the pathogenesis of Costello syndrome, J. Hum. Genet., 56, 707-715 (2011).

46. Rangarajan A, Weinberg RA, Comparative biology of mouse versus human cells: modelling human cancer in mice, Nat. Rev. Cancer, 12, 952-959 (2003).

47. Tuveson DA, Shaw AT, Willis NA, Silver DP, Jackson EL, Chang $S$, et al., Endogenous oncogenic K-ras G12D stimulates proliferation and widespread neoplastic and developmental defects, Cancer Cell, 4, 375-377 (2004).

48. Aravinthan A, Shannon N, Heaney J, Hoare M, Marshall A, Alexander GJM, The senescent hepatocyte gene signature in chronic liver disease, Exp. Gerontol., 60, 37-45 (2014).
Supplementary Fig. 1. - Erk1/2 and Akt activation in $\mathrm{CS}$ adult mouse skin fibroblasts (ASFs). Immunoblotting analysis (three independent experiments) of cleared cell lysates was performed using specific antibodies against total and phospho-proteins. Gapdh was used as the control for protein normalization 


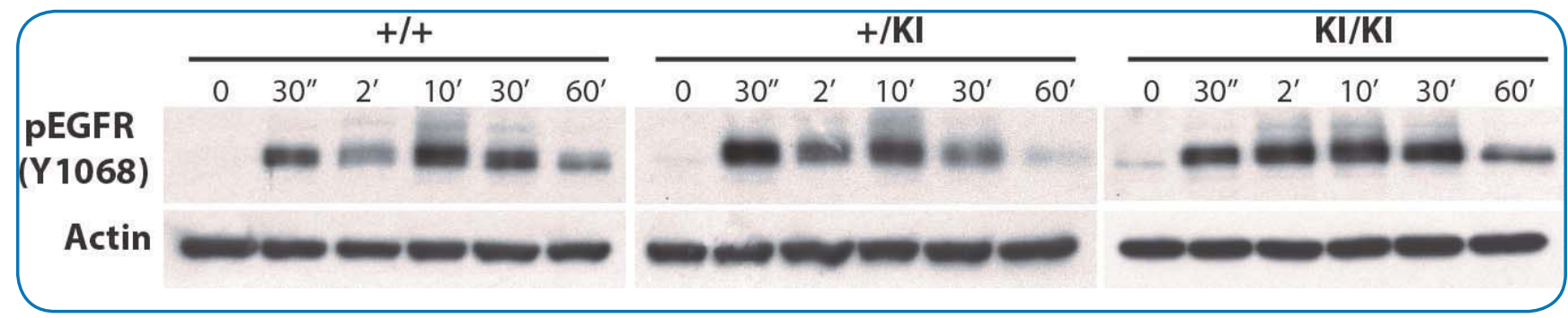

Supplementary Fig. 2. - Representative image of Egfr activation in MEFs. Egfr activation was monitored via immunoblotting analysis using phosphorylated Egfr (Y1068) antibody (Cell Signaling).

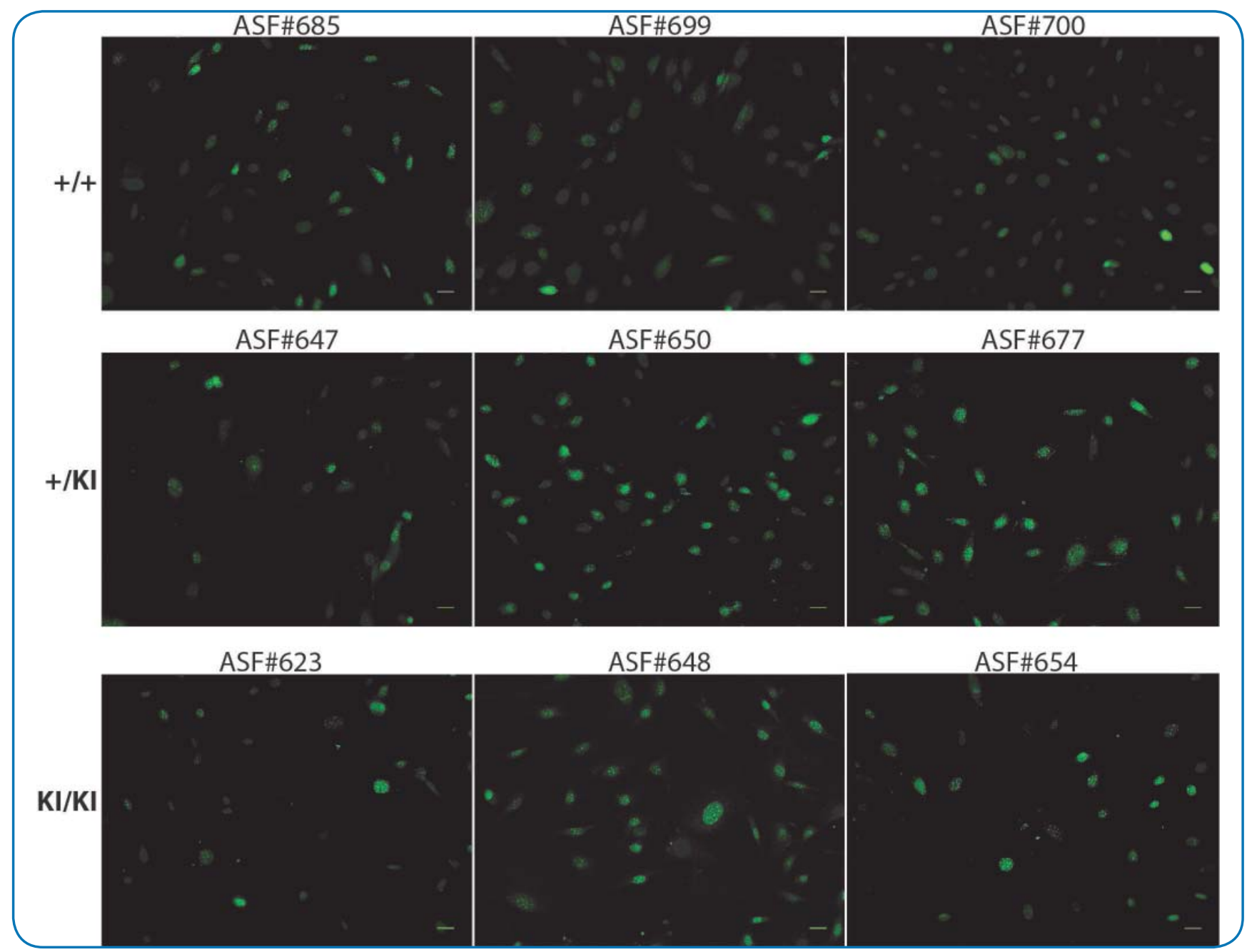

Supplementary Fig. 3. - Immunofluorescence analysis of senescence-associated heterochromatin foci (SAHF) detected using HP1 $a$ antibodies (green). ASFs numbers represent different mice used for isolating skin fibroblasts. Images were acquired using Apotome 2 fluorescence microscope (Zeiss). ASFs 700 (wild type), 647 (+/KI) and 623 (KI/KI) were used in the Figure 3d. Scale bar $20 \mu \mathrm{m}$.

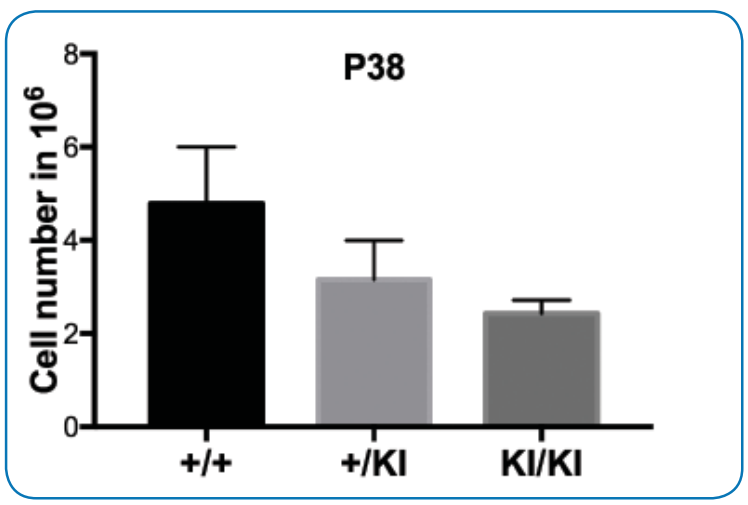

Supplementary Fig. 4. - Reduction of cell number at P38 in CS ASFs. Cells were trypsinized, counted using Neubauer chamber and the numbers were plotted using Prism 6 (GraphPad). 